\title{
Current constraints on early dark energy and growth index using latest observations
}

\author{
F. Y. Wang ${ }^{1,2}$ \\ 1 School of Astronomy and Space Science, Nanjing University, 210093 Nanjing, PR China \\ e-mail: fayinwang@nju.edu.cn \\ ${ }^{2}$ Key laboratory of Modern Astronomy and Astrophysics (Nanjing University), Ministry of Education, 210093 Nanjing, PR China \\ Received 5 April 2012 / Accepted 18 May 2012
}

\begin{abstract}
Aims. In this paper we study observational constraints on early dark energy model proposed by Doran \& Robbers and growth index using the latest Union2 type Ia supernovae (SNe Ia), the baryon acoustic oscillations (BAO) in the large-scale correlation function of the Sloan Digital Sky Survey and Two-degree Field Galaxy Redshift Survey, cosmic microwave background (CMB) from the Wilkinson Microwave Anisotropy Probe seven-year data, the linear growth factors data and gamma-ray bursts (GRBs).

Methods. By using the $\chi^{2}$ statics method, we constrain the early dark energy model and growth factor from the above datasets. When including the GRB data, we use the cosmographic parameters to calibrate the GRB luminosity relations using SNe Ia.

Results. At $2 \sigma$ confidence level, we find that the fractional dark energy density at early times is $\Omega_{\mathrm{d}}^{\mathrm{e}}<0.05 \mathrm{using} \mathrm{SNe}$ Ia, CMB and BAO. When we include high-redshift probes, such as measurements of the linear growth factors and GRBs, the constraint is tightened considerably and becomes $\Omega_{\mathrm{d}}^{\mathrm{e}}<0.03$ at $2 \sigma$ confidence level. We also discuss the growth rate index $\gamma$. We find $\gamma=$ $0.661_{-0.203}^{+0.302}(1 \sigma)$ using the SNe Ia, CMB, BAO and linear growth factor data. After including high-redshift GRB data, the growth index is $\gamma=0.653_{-0.363}^{+0.372}$.
\end{abstract}

Key words. dark energy - cosmological parameters

\section{Introduction}

Current cosmological observations reveal that the Universe is now undergoing an accelerated phase of expansion and dark energy comprises roughly $70 \%$ of the energy density of our Universe (Riess et al. 1998; Perlmutter et al. 1999). The nature of dark energy is one of the most important problems in modern physics and has been extensively investigated. A possible candidate responsible for this component is the usual vacuum energy represented by a cosmological constant $\Lambda$ which has a negative pressure (Weinberg 1989; Peebles \& Ratra 2003). However, it requires fine tuning to make the cosmological constant energy density dominant at the recent epoch. Many dynamical dark energy models also have been proposed in the literature, e.g., quintessence (Wetterich 1988; Ratra \& Peebles 1988), phantom (Caldwell 2002), quintom (Feng et al. 2005).

However, in dynamic models of dark energy, such as a scalar field, this may be different and dark energy could have a nonnegligible influence on earlier stages of the growth of cosmic structures. Non-negligible dark energy density at high redshifts would indicate dark energy physics distinct from a cosmological constant or reasonable scalar fields. Such dark energy in the dark ages can be constrained tightly through investigation of the growth of structure and other high-redshift probes, such as gamma-ray bursts (GRBs). These models are known as early dark energy (EDE) models.

Many early dark energy models have been proposed (Wetterich 2004; Linder 2006; Doran \& Robbers 2006) and its effect on cosmological structure is discussed extensively (Francis et al. 2008; Mota 2008; Grossi \& Springel 2009). By combining cosmic microwave background (CMB), large scale structure and type Ia supernovae data, Doran \& Robbers (2006) constrained the fraction of EDE density $\Omega_{\mathrm{d}}^{\mathrm{e}}<0.06$ (Doran \& Robbers 2006). Linder \& Robbers confirmed the relevance of early dark energy on the CMB power spectrum if $\Omega_{\mathrm{d}}^{\mathrm{e}}$ is larger than 0.03 , and emphasized that ignoring early dark energy can severely bias the determination of the equation of state (EOS) from baryon acoustic oscillations (BAO, Linder \& Robbers 2008). Xia \& Viel also constrain the parameters of one EDE parameterization using more high-redshift data (Xia \& Viel 2009). de Putter et al. (2009) and Hollenstein et al. (2009) used the lensed CMB temperature and polarization power spectra to constrain early dark energy at high redshifts (de Putter et al. 2009; Hollenstein et al. 2009). The early dark energy model including dark energy perturbations was also studied by (Alam 2010; Alam et al. 2011). The constraints from future data such as CMB was also extensively discussed in (Calabrese et al. 2011a,b). Reichardt et al. (2012) used CMB data from Wilkinson Microwave Anisotropy Probe (WMAP) and South Pole Telescope to give new limits on early dark energy.

In this paper, we constrain early dark energy model proposed by Doran \& Robbers (2006) using latest Union2 sample type Ia supernovae (SNe Ia, Amanullah et al. 2010), the seven-year Wilkinson Microwave Anisotropy Probe (WMAP7) observations (Komatsu et al. 2011), and the baryon acoustic oscillation (BAO) measurement from the Sloan Digital Sky Survey (SDSS), the Two-degree Field Galaxy Redshift Survey (2dFGRS) (Percival et al. 2010), the linear growth factors data and GRBs (Wang et al. 2011). For the GRBs, we use the latest GRB sample that includes 116 GRBs. We also investigate the influence on growth history at high-redshift by early dark energy and constrain the growth factor using observational datasets. 


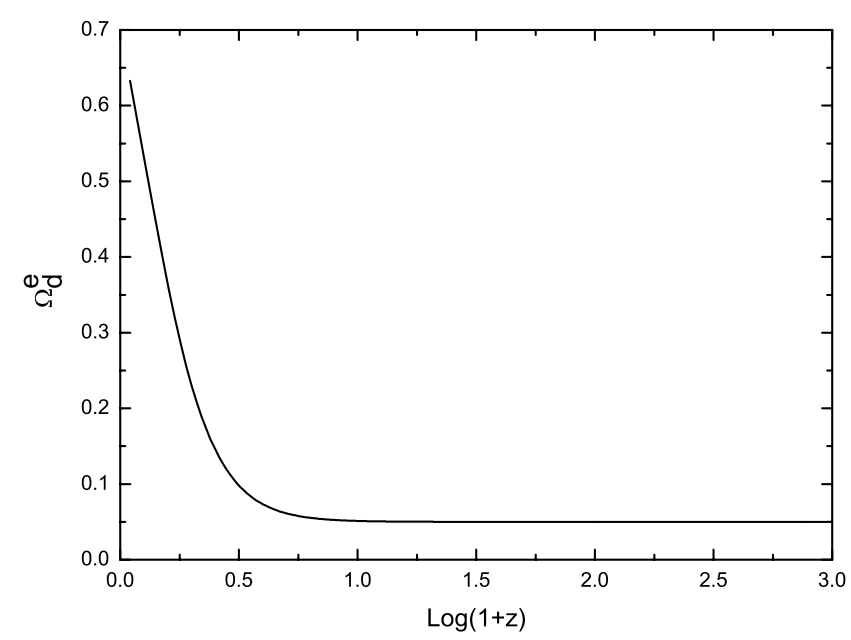

Fig. 1. The early dark energy density $\Omega_{\mathrm{d}}^{\mathrm{e}}$ as a function of redshift with $\Omega_{\mathrm{d}}^{\mathrm{e}}=0.03$.

The structure of this paper is as follows. In Sect. 2, we give the basic equations of early dark energy models. Observational data and analysis method are shown in Sect. 3. In Sect. 4, we show the results on early dark energy model from the current observations. Section 5 contains constraints on growth factor. Conclusions and discussion are presented in Sect. 6.

\section{The basic equations of early dark energy models}

We consider the early dark energy model introduced in Doran \& Robbers (2006). This method is directly to parameterize the dark energy density. This will prove advantageous for two reasons: firstly, the amount of dark energy at early times is then a natural parameter and not inferred by integrating $w(a)$ over the entire evolution. Secondly, since the Hubble parameter is given by

$\frac{H^{2}(a)}{H_{0}^{2}}=\frac{\Omega_{\mathrm{m}} a^{-3}+\Omega_{\mathrm{rel}} a^{-4}}{1-\Omega_{\mathrm{d}}(a)}$,

a simple, analytic expression for $\Omega_{\mathrm{d}}(a)$ enables us to compute many astrophysical quantities analytically. Here $\Omega_{\text {rel }}^{0}$ is the fractional energy density of relativistic neutrinos and photons today, $\Omega_{\mathrm{m}}^{0}$ is the matter fractional energy density.

The form of parameterization, namely

$\Omega_{\mathrm{d}}(a)=\frac{\Omega_{\mathrm{d}}-\Omega_{\mathrm{d}}^{\mathrm{e}}\left(1-a^{-3 w_{0}}\right)}{\Omega_{\mathrm{d}}+\Omega_{\mathrm{m}} a^{3 w_{0}}}+\Omega_{\mathrm{d}}^{\mathrm{e}}\left(1-a^{-3 w_{0}}\right)$.

Here $\Omega_{\mathrm{d}}=1-\Omega_{\mathrm{m}}$ is the present dark energy density, $\Omega_{\mathrm{d}}^{\mathrm{e}}$ is the asymptotic early dark energy density, and $w_{0}$ is the present dark energy equation of state (EOS). In addition to the matter density, the two parameters are $\Omega_{\mathrm{d}}^{\mathrm{e}}$ and $w_{0}$. The standard formula for the EOS, $w=-1 /\left(3\left[1-\Omega_{\mathrm{d}}(a)\right]\right) \mathrm{d} \ln \Omega_{\mathrm{d}}(a) / \mathrm{d} \ln a$, can not be simplified in this model. The deceleration parameter can be derived by, $q(z)=(1+z) H^{-1} \mathrm{~d} H / \mathrm{d} z-1$.

In Fig. 1 we plot the dark energy density as a function of redshift with $\Omega_{\mathrm{m}}^{0}=0.30, w_{0}=-1.08$ and $\Omega_{\mathrm{d}}^{\mathrm{e}}=0.03$. In Fig. 2 we show the equation of state as a function of redshift. We can see that the dark energy density does not act to accelerate expansion at early times, and in fact $w \rightarrow 0$. In Fig. 3, we plot the deceleration parameter $q(z)$ as a function of redshift. The transition redshift is $z_{\mathrm{T}}=0.62$.

The growth of perturbations is strongly affected by the unclustered early dark energy. We introduce the linear perturbation

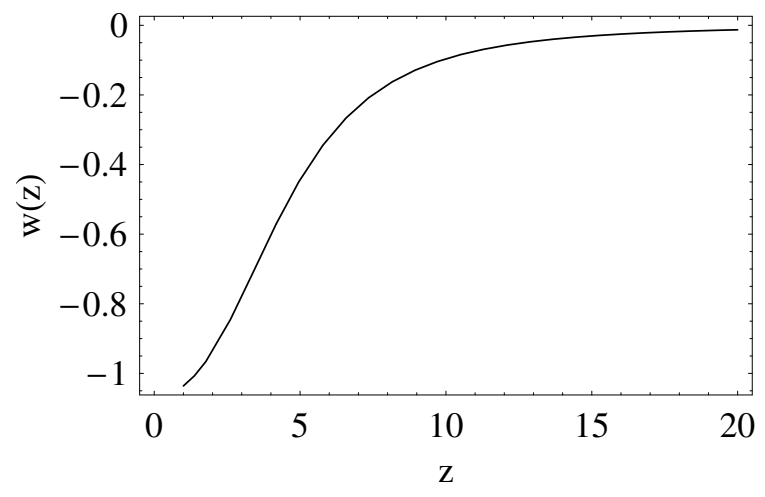

Fig. 2. The equation of state $w(z)$ of early dark energy as a function of redshift with $\Omega_{\mathrm{d}}^{\mathrm{e}}=0.03$.

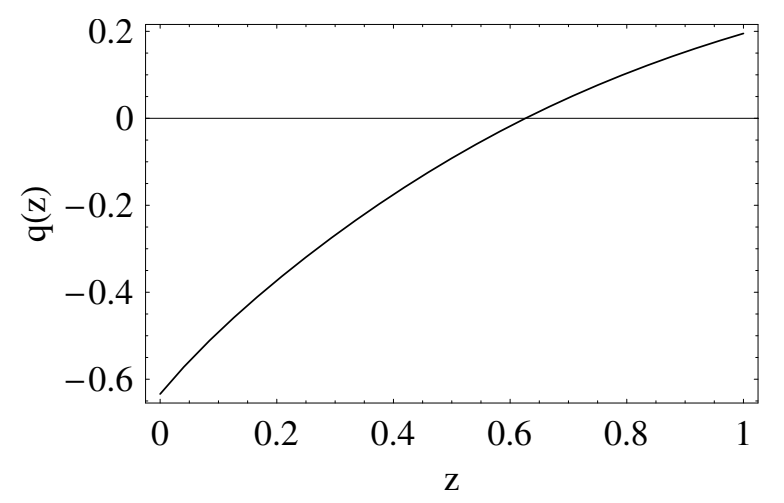

Fig. 3. The evolution of deceleration parameter $q(z)$ with $\Omega_{\mathrm{d}}^{\mathrm{e}}=0.03$. The horizontal line shows $q(z)=0$.

growth theory as follow. To linear order of perturbation, at large scales the matter density perturbation $\delta=\delta \rho_{\mathrm{m}} / \rho_{\mathrm{m}}$ satisfies the simple equation:

$\ddot{\delta}+2 H \dot{\delta}-4 \pi G \rho_{\mathrm{m}} \delta=0$,

where $\rho_{\mathrm{m}}$ is the matter energy density. In terms of the growth factor $f=\mathrm{d} \ln \delta / \mathrm{d} \ln a$, the matter density perturbation Eq. (3) becomes

$f^{\prime}+f^{2}+\left(\frac{\dot{H}}{H^{2}}+2\right) f=\frac{3}{2} \Omega_{\mathrm{m}}$,

where $f^{\prime}=\mathrm{d} f / \mathrm{d} \ln a$. The solution of the equation can be approximated as $f=\Omega_{\mathrm{m}}^{\gamma}$ (Peebles 1980; Fry 1985; Lightman \& Schechter 1990; Wang \& Steinhardt 1998) and the growth index $\gamma$ can be obtained for some general models. For a dynamical dark energy model with slowly varying $w$ and zero curvature, the approximation $f(z)=\Omega(z)^{\gamma}$ was given in Wang \& Steinhardt (1998). For more general dynamical dark energy models in flat space, it was found that $\gamma=0.55+0.05[1+w(z=1)]$ with $w>-1$ and $\gamma=0.55+0.02[1+w(z=1)]$ with $w<-1$ (Linder 2005; Linder \& Cahn 2007). For the flat DGP model, $\gamma=11 / 16$ (Linder \& Cahn 2007). In non-flat case, an approximation for the growth factor $f=\Omega_{\mathrm{m}}^{\gamma}+(\gamma-4 / 7) \Omega_{k}$ is given in Gong et al. (2009). More recently Linder (2009) shows that the numerical solution of Eq. (4) can be approximated as

$g(z) \equiv \frac{\delta(z)}{\delta(0)}=g_{\star} e^{\int_{1}^{\frac{1}{1+z}} \Omega_{\mathrm{m}}(a)^{\gamma} \frac{\mathrm{d} a}{a}}$

where $g_{\star}$ is the calibration parameter of the growth behavior at early times. Enhanced growth involves $g_{\star}>1$, and any deviation from $g_{\star}=1$ signals a nonstandard early universe behavior (Linder 2009). The calibration parameter is important in 
F. Y. Wang: Constraints on EDE and growth index

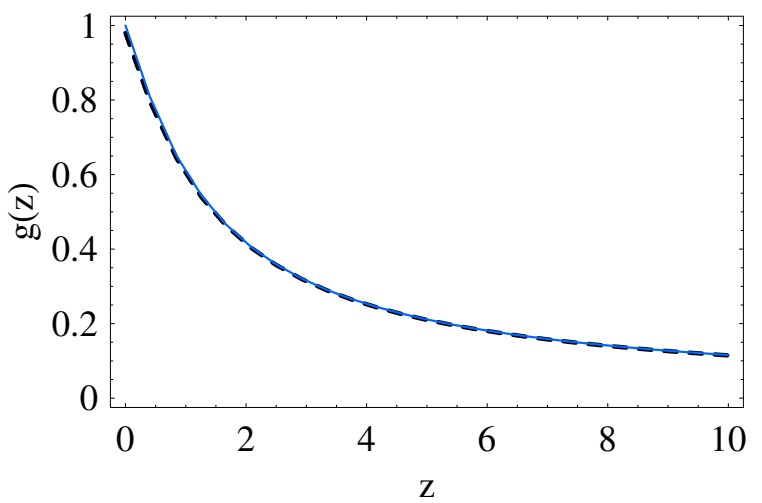

Fig. 4. The numerically obtained solution of Eq. (3) and the normalized growth of Eq. (5). The dashed line shows the numerical result of Eq. (3) and the solid line shows result from Eq. (5) with $\Omega_{\mathrm{m}}^{0}=0.29, w_{0}=$ $-1.04, \Omega_{\mathrm{d}}^{\mathrm{e}}=0.03, g_{\star}=0.98$ and $\gamma=6 / 11$.

early dark energy cosmology. In Fig. 4, we show the normalized growth of Eq. (5) and the numerical result of Eq. (3). The dashed line shows the numerical result of Eq. (3) and the solid line shows result from Eq. (5) with $\Omega_{\mathrm{m}}^{0}=0.29, w_{0}=-1.04$, $\Omega_{\mathrm{d}}^{\mathrm{e}}=0.03, g_{\star}=0.98$ and $\gamma=6 / 11$. These parameter are obtained from latest observational data constraint. We show that the numerical solution of Eq. (4) is well consistent with the approximate result (Eq. (5)).

\section{Observational data and analysis methods}

\subsection{Type la supernovae (SNe la)}

First, we use the Union2 compilation of 557 type Ia SNe (Amanullah et al. 2010). The theoretical distance modulus is defined as

$\mu_{\mathrm{th}}\left(z_{i}\right) \equiv 5 \log _{10} d_{\mathrm{L}}\left(z_{i}\right)+\mu_{0}$

where $\mu_{0} \equiv 42.38-5 \log _{10} h$ with $h$ is the Hubble constant $H_{0}$ in units of $100 \mathrm{~km} / \mathrm{s} / \mathrm{Mpc}$, and

$d_{\mathrm{L}}(z)=(1+z) \int_{0}^{z} \frac{\mathrm{d} z^{\prime}}{E\left(z^{\prime} ; \theta\right)}$

is the Hubble-free luminosity distance $H_{0} d_{\mathrm{L}}$ (here $d_{\mathrm{L}}$ is the physical luminosity distance) in a spatially flat FRW universe, and here $\theta$ denotes the model parameters. The $\chi^{2}$ for the $\mathrm{SNe}$ Ia data is

$\chi_{\mathrm{SN}}^{2}(\theta)=\sum_{i=1}^{557} \frac{\left[\mu_{\mathrm{obs}}\left(z_{i}\right)-\mu_{\mathrm{th}}\left(z_{i}\right)\right]^{2}}{\sigma_{i}^{2}}$,

where $\mu_{\mathrm{obs}}\left(z_{i}\right)$ and $\sigma_{i}$ are the observed value and the corresponding $1 \sigma$ error of distance modulus for each supernova, respectively. The parameter $\mu_{0}$ is a nuisance parameter but it is independent of the data and the dataset. Following Nesseris \& Perivolaropoulos (2005), the minimization with respect to $\mu_{0}$ can be made trivially by expanding the $\chi^{2}$ of Eq. (8) with respect to $\mu_{0}$ as

$\chi_{\mathrm{SN}}^{2}(\theta)=A(\theta)-2 \mu_{0} B(\theta)+\mu_{0}^{2} C$, where

$$
\begin{aligned}
A(\theta) & =\sum_{i=1}^{557} \frac{\left[\mu_{\mathrm{obs}}\left(z_{i}\right)-\mu_{\mathrm{th}}\left(z_{i} ; \mu_{0}=0, \theta\right)\right]^{2}}{\sigma_{i}^{2}}, \\
B(\theta) & =\sum_{i=1}^{557} \frac{\mu_{\mathrm{obs}}\left(z_{i}\right)-\mu_{\mathrm{th}}\left(z_{i} ; \mu_{0}=0, \theta\right)}{\sigma_{i}^{2}}, \\
C & =\sum_{i=1}^{557} \frac{1}{\sigma_{i}^{2}} .
\end{aligned}
$$

Evidently, Eq. (8) has a minimum for $\mu_{0}=B / C$ at

$\tilde{\chi}_{\mathrm{SN}}^{2}(\theta)=A(\theta)-\frac{B(\theta)^{2}}{C}$.

Since $\chi_{\mathrm{SN}, \mathrm{min}}^{2}=\tilde{\chi}_{\mathrm{SN}, \mathrm{min}}^{2}$, instead minimizing $\chi_{\mathrm{SN}}^{2}$ one can minimize $\tilde{\chi}_{\mathrm{SN}}^{2}$ which is independent of the nuisance parameter $\mu_{0}$.

\subsection{Cosmic microwave background}

In order to implement the WMAP7 data, we use the distance priors from (Komatsu et al. 2011). It has been shown that the constraints on cosmological parameters from WMAP7 distance priors are good agreement with the full MCMC results (Komatsu et al. 2011). We use three fitting parameters $R, l_{\mathrm{a}}$ and $z_{*}$. The acoustic scale $l_{\mathrm{a}}$ is

$l_{\mathrm{a}}=\frac{\pi d_{\mathrm{L}}\left(z_{*}\right)}{\left(1+z_{*}\right) r_{\mathrm{s}}\left(z_{*}\right)}=302.09 \pm 0.76$,

where the redshift $z_{*}$ is given by (Hu \& Sugiyama 1996)

$$
\begin{aligned}
z_{*} & =1048\left[1+0.00124\left(\Omega_{\mathrm{b}} h^{2}\right)^{-0.738}\right]\left[1+g_{1}\left(\Omega_{\mathrm{m}} h^{2}\right)^{g_{2}}\right] \\
& =1091.3 \pm 0.91 \\
g_{1} & =\frac{0.0783\left(\Omega_{\mathrm{b}} h^{2}\right)^{-0.238}}{1+39.5\left(\Omega_{\mathrm{b}} h^{2}\right)^{0.763}}, \quad g_{2}=\frac{0.560}{1+21.1\left(\Omega_{\mathrm{b}} h^{2}\right)^{1.81}},
\end{aligned}
$$

and $r_{\mathrm{s}}\left(z_{*}\right)$ is the comoving sound horizon at $z_{*}$. We must notice that comoving sound horizon changes as $r_{\mathrm{s}}\left(z, \Omega_{\mathrm{d}}^{\mathrm{e}}\right)=r_{\mathrm{s}}\left(z, \Omega_{\mathrm{d}}^{\mathrm{e}}=\right.$ 0) $\sqrt{1-\Omega_{\mathrm{d}}^{\mathrm{e}}}$. The shift parameter

$R=\sqrt{\Omega_{\mathrm{m}}} \int_{0}^{z_{*}} \frac{\mathrm{d} z}{E(z)}=1.725 \pm 0.018$

The $\chi^{2}$ of the CMB data is constructed as $\chi_{\mathrm{CMB}}^{2}=X^{T} C_{\mathrm{CMB}}^{-1} X$, where

$X=\left(\begin{array}{l}l_{A}-302.09 \\ R-1.725 \\ z_{*}-1091.3\end{array}\right)$,

and the inverse covariance matrix is given by (Komatsu et al. 2011)

$C_{\mathrm{CMB}}^{-1}=\left(\begin{array}{ccc}2.305 & 29.698 & -1.333 \\ 29.698 & 6825.270 & -113.180 \\ -1.333 & -113.180 & 3.414\end{array}\right)$. 


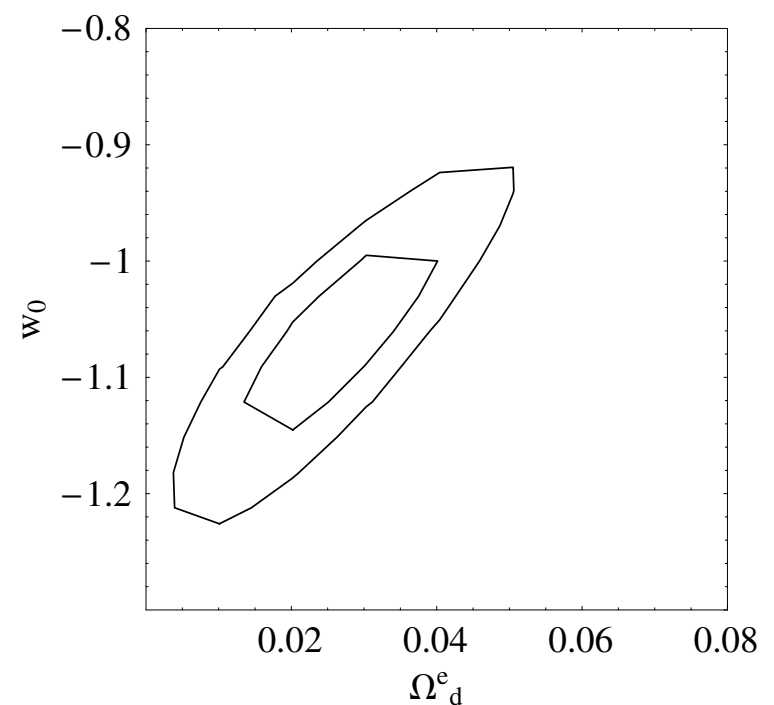

Fig. 5. Constraints on $\Omega_{\mathrm{d}}^{\mathrm{e}}$ and $w_{0}$ from $1 \sigma$ to $2 \sigma$ using SNe Ia, CMB and $\mathrm{BAO}$.

\subsection{Baryon acoustic peak from SDSS and 2dFGRS}

It is well known that the acoustic peaks in CMB anisotropy power spectrum can be used to determine the properties of perturbations and to constrain cosmological parameters and dark energy. The acoustic peaks occur because the cosmic perturbations excite sound waves in the relativistic plasma of the early universe. Because the universe has a fraction of baryons, the acoustic oscillations in the relativistic plasma will be imprinted onto the late-time power spectrum of the nonrelativistic matter (Eisenstein \& Hu 1998). The acoustic signatures in the largescale clustering of galaxies can also be used to constrain cosmological parameters and dark energy. The detection of a peak in the correlation function of luminous red galaxies in the 2dFGRS and Sloan Digital Sky Survey. This peak can provide a"standard ruler" with which to constrain cosmological parameters.

To use the baryon acoustic oscillations (BAO) measurement from the SDSS and 2dFGRS data, we define (Percival et al. 2010)

$\boldsymbol{X}_{\mathrm{BAO}}=\left(\begin{array}{c}r_{\mathrm{s}}\left(z_{\mathrm{d}}\right) / D_{V}(0.2)-0.1905 \\ r_{\mathrm{s}}\left(z_{\mathrm{d}}\right) / D_{V}(0.35)-0.1097\end{array}\right)$

and using the inverse covariance matrix (Percival et al. 2010)

$\mathbf{C}_{\mathrm{BAO}}{ }^{-1}=\left(\begin{array}{cc}30124 & -17227 \\ -17227 & 86977\end{array}\right)$,

we find the contribution of BAO to $\chi^{2}$ as

$\chi_{\mathrm{BAO}}^{2}=\boldsymbol{X}_{\mathrm{BAO}}{ }^{\mathrm{T}} \mathbf{C}_{\mathrm{BAO}}{ }^{-1} \boldsymbol{X}_{\mathrm{BAO}}$,

where the effective distance is

$D_{V}(z)=\left[\frac{d_{\mathrm{L}}^{2}(z)}{(1+z)^{2}} \frac{z}{H(z)}\right]^{1 / 3}$.

The effect of early dark energy is a change in $D_{V}(z)$, such as $D_{V}\left(z=0.2, \Omega_{\mathrm{d}}^{\mathrm{e}}\right)=D_{V}\left(z=0.2, \Omega_{\mathrm{d}}^{\mathrm{e}}=0\right) \sqrt{1-\Omega_{\mathrm{d}}^{\mathrm{e}}}$ and $D_{V}\left(z=0.35, \Omega_{\mathrm{d}}^{\mathrm{e}}\right)=D_{V}\left(z=0.35, \Omega_{\mathrm{d}}^{\mathrm{e}}=0\right) \sqrt{1-\Omega_{\mathrm{d}}^{\mathrm{e}}}$ (Doran et al. 2007). The redshift $z_{\mathrm{d}}$ is fitted with the formulae (Eisenstein \& Hu 1998)

$z_{\mathrm{d}}=\frac{1291\left(\Omega_{\mathrm{m}} h^{2}\right)^{0.251}}{1+0.659\left(\Omega_{\mathrm{m}} h^{2}\right)^{0.828}}\left[1+b_{1}\left(\Omega_{\mathrm{b}} h^{2}\right)^{b_{2}}\right]$,

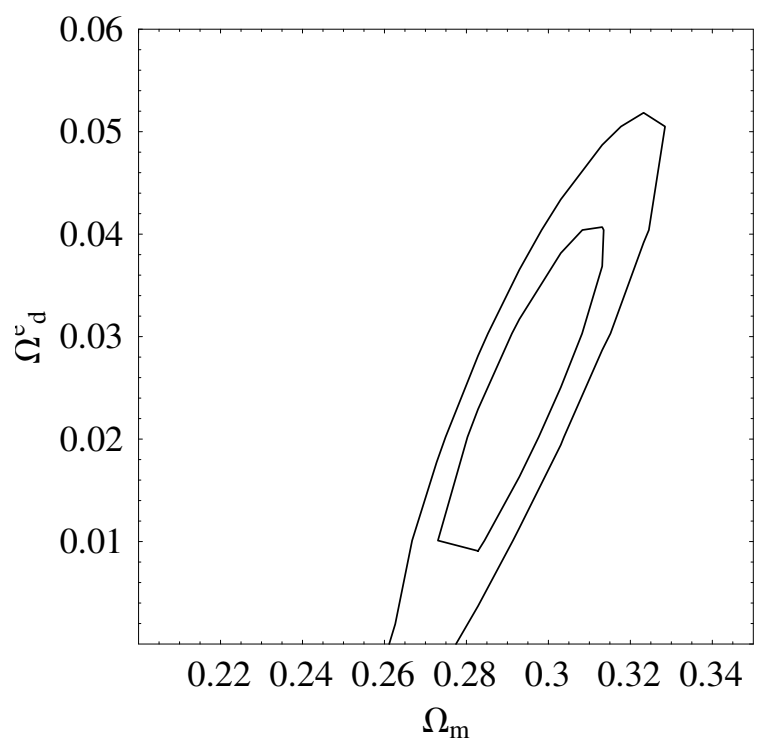

Fig. 6. The $1 \sigma, 2 \sigma$ confidence regions for $\Omega_{\mathrm{m}}$ versus $\Omega_{\mathrm{d}}^{\mathrm{e}}$ derived using $\mathrm{SNe} \mathrm{Ia}, \mathrm{CMB}$ and BAO.

$b_{1}=0.313\left(\Omega_{\mathrm{m}} h^{2}\right)^{-0.419}\left[1+0.607\left(\Omega_{\mathrm{m}} h^{2}\right)^{0.674}\right]$,
$b_{2}=0.238\left(\Omega_{\mathrm{m}} h^{2}\right)^{0.223}$

and the comoving sound horizon is

$r_{\mathrm{S}}(z)=\int_{0}^{\infty} \frac{\mathrm{d} z^{\prime}}{c_{\mathrm{s}}\left(z^{\prime}\right) E\left(z^{\prime}\right)}$,

where the sound speed $\left.c_{\mathrm{s}}(z)=1 / \sqrt{3\left[1+\overline{R_{\mathrm{b}}} /(1+z)\right.}\right], \overline{R_{\mathrm{b}}}=$ $315000 \Omega_{\mathrm{b}} h^{2}\left(T_{\mathrm{cmb}} / 2.7 \mathrm{~K}\right)^{-4}$. The CMB temperature is $T_{\mathrm{cmb}}=$ $2.725 \mathrm{~K}$.

\section{Observational constraints on early dark energy}

For Gaussian distributed measurements, the likelihood function $L \propto e^{-\chi^{2} / 2}$, with

$\chi^{2}=\chi_{\mathrm{SN}}^{2}+\chi_{\mathrm{CMB}}^{2}+\chi_{\mathrm{BAO}}^{2}$.

We also marginalize nuisance parameters $H_{0}$ and $\Omega_{\mathrm{b}} h^{2}$. The latest Hubble constant is $H_{0}=74.2 \pm 3.6 \mathrm{~km} \mathrm{~s}^{-1} \mathrm{Mpc}$ (Riess et al. 2009). The baryon density is $\Omega_{\mathrm{b}} h^{2}=0.02265 \pm 0.00059$ (Komatsu et al. 2011). The likelihood for the parameters $\left(\Omega_{\mathrm{m}}\right.$, $w_{0}, \Omega_{\mathrm{d}}^{\mathrm{e}}$ ) in the model is computed by minimizing the $\chi^{2}$. Our program randomly chooses values for the above parameters, evaluate $\chi^{2}$.

By fitting the early dark energy model using combined data, we find $\chi_{\min }^{2}=552.93, \Omega_{\mathrm{m}}=0.287_{-0.019}^{+0.021}, \Omega_{\mathrm{d}}^{\mathrm{e}}=0.027_{-0.017}^{+0.010}$, and $w_{0}=-1.054_{-0.072}^{+0.043}$. Figure 5 shows constraints on the $\Omega_{\mathrm{d}}^{\mathrm{e}}-w_{0}$ plane. Figure 6 shows constraints on the $\Omega_{\mathrm{m}}-\Omega_{\mathrm{d}}^{\mathrm{e}}$ plane. At $2 \sigma$ confidence level, we find that the fractional dark energy density $\Omega_{\mathrm{d}}^{\mathrm{e}}<0.05$.

\subsection{High-redshift probes}

In order to make tight constraints on early dark energy models, we must add high-redshift probes, such as GRBs and linear growth factors data. GRBs can be detectable out to very high redshifts. The farthest burst detected so far is GRB 090429B, which is at $z=9.4$ (Cucchiara et al. 2011). GRBs could in 
Table 1. $\sigma_{8}$ values and growth rates with $1 \sigma$ error bars from which we derived the linear growth factors used in our analysis.

\begin{tabular}{lccc}
\hline \hline$z$ & $\sigma_{8}$ & $\sigma_{\sigma_{8}}$ & Ref. \\
\hline 2.125 & 0.95 & 0.17 & (Viel et al. 2004) \\
2.72 & 0.92 & 0.17 & (Viel \& Haehnelt 2006) \\
\hline 2.2 & 0.92 & 0.16 & \\
2.4 & 0.89 & 0.11 & \\
2.6 & 0.98 & 0.13 & \\
2.8 & 1.02 & 0.09 & \\
3.0 & 0.94 & 0.08 & \\
3.2 & 0.88 & 0.09 & \\
3.4 & 0.87 & 0.12 & Ref. \\
3.6 & 0.95 & 0.16 & \\
3.8 & 0.90 & 0.17 & (Tegmark et al. 2006) \\
\hline$z$ & $f_{\text {obs }}$ & $\sigma_{f_{\text {obs }}}$ & (Ross et al. 2007) \\
0.15 & 0.51 & 0.11 & (Hawkins et al. 2003; Verde et al. 2002) \\
0.35 & 0.70 & 0.18 & (da Angela et al. 2008) \\
0.55 & 0.75 & 0.18 & (McDonald et al. 2005) \\
1.40 & 0.90 & 0.24 & Ref. \\
3.00 & 1.46 & 0.29 & (Blake et al. 2011) \\
\hline$z$ & $f_{\text {obs }} \sigma_{8}$ & $\sigma_{f_{\text {obs }} \sigma_{8}}$ & (Blake et al. 2011) \\
0.22 & 0.60 & 0.10 & (Blake et al. 2011) 2011) \\
0.41 & 0.70 & 0.07 & \\
0.60 & 0.73 & 0.07 & \\
0.78 & 0.70 & 0.08 &
\end{tabular}

principle serve as such high redshift standardizable candles (Dai et al. 2004; Wang 2008; Wang et al. 2007, 2009; Basilakos \& Perivolaropoulos 2008; Izzo et al. 2009; Schaefer 2007). We use the latest 116 GRBs sample summarized in Wang et al. (2011). We use the method shown in Wang \& Dai (2011) to calibrate the GRB luminosity relations. First we fit the cosmographic parameters using the Union2 sample. Then we deduce the distance moduli of GRBs at $z \leq 1.4$. Last using these deduced distance moduli and the redshifts of corresponding GRBs, we calibrate four GRB luminosity correlations. We assume these relations do not evolve with redshift and are valid at $z>1.40$, which is also confirmed by Wang et al. (2011). The luminosity or energy of GRB can be calculated. So the luminosity distances and distance modulus can be obtained. After obtaining the distance modulus of each burst using one of these relations, we use the same method as Schaefer (2007) to calculate the real distance modulus,

$\mu_{\mathrm{fit}}=\left(\sum_{i} \mu_{i} / \sigma_{\mu_{i}}^{2}\right) /\left(\sum_{i} \sigma_{\mu_{i}}^{-2}\right)$,

where the summation runs from 1-4 over the relations with available data, $\mu_{i}$ is the best estimated distance modulus from the $i$ th relation, and $\sigma_{\mu_{i}}$ is the corresponding uncertainty. The uncertainty of the distance modulus for each burst is

$\sigma_{\mu_{\mathrm{fit}}}=\left(\sum_{i} \sigma_{\mu_{i}}^{-2}\right)^{-1 / 2}$.

The $\chi^{2}$ value is

$\chi_{\mathrm{GRB}}^{2}=\sum_{i=1}^{N} \frac{\left[\mu_{i}\left(z_{i}\right)-\mu_{f i t, i}\right]^{2}}{\sigma_{\mu_{\mathrm{fit}, i}}^{2}}$,

where $\mu_{\mathrm{fit}, i}$ and $\sigma_{\mu_{\mathrm{fit}, i}}$ are the fitted distance modulus and its error. This method is almost model-independent.

We also use the linear growth factor data in Table 1. There are 20 data points at a redshift range $(0.15,3.8)$ in Table 1 . We

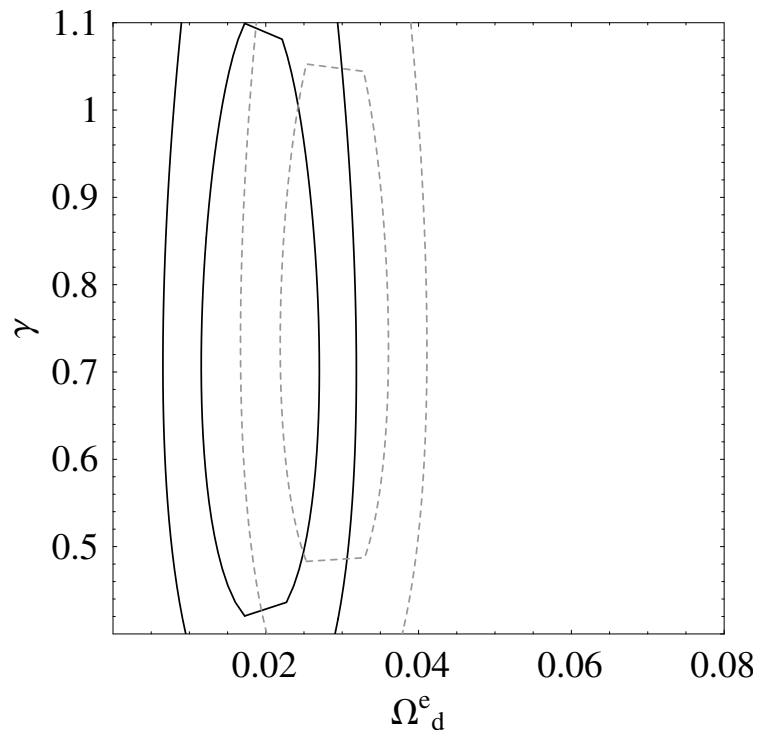

Fig. 7. Solid contours: the $1 \sigma, 2 \sigma$ confidence regions for $\gamma$ versus $\Omega_{d}^{e}$ derived using $\mathrm{SNe}$ Ia, CMB, BAO, GRBs and the linear growth factor data. Dashed contours: the $1 \sigma, 2 \sigma$ confidence regions for $\gamma$ versus $\Omega_{\mathrm{d}}^{\mathrm{e}}$ derived using $\mathrm{SNe} \mathrm{Ia}, \mathrm{CMB}$ and $\mathrm{BAO}$.

include the growth rate data from WiggleZ Dark Energy Survey (Blake et al. 2011). Balke et al. (2011) presented fits for the growth rate of structure using redshift-space distortions in the $2 \mathrm{D}$ power spectrum. These fits included a full exploration of the systematic errors arising from the assumption of redshift-space distortion models based on perturbation theory techniques, fitting formulae calibrated by N-body simulations, and empirical models. After including GRBs and linear growth factor data, we find $\Omega_{\mathrm{m}}=0.303_{-0.018}^{+0.017}, \Omega_{\mathrm{d}}^{\mathrm{e}}=0.019_{-0.015}^{+0.010}$, and $w_{0}=-1.075_{-0.078}^{+0.035}$ with $\chi_{\min }^{2}=630.68$. In Fig. 7, we show the confidence regions for $\gamma$ and $\Omega_{\mathrm{d}}^{\mathrm{e}}$ from $1 \sigma$ to $2 \sigma$. Dotted contours show constraints from $\mathrm{SN}+\mathrm{CMB}+\mathrm{BAO}$. The solid contours are obtained by adding GRBs and linear growth factors data. By minimizing $\chi^{2}=\chi_{\mathrm{SN}}^{2}+\chi_{\mathrm{CMB}}^{2}+\chi_{\mathrm{BAO}}^{2}+\chi_{\gamma}^{2}$, we find $\Omega_{\mathrm{d}}^{\mathrm{e}}<0.04$ at $2 \sigma$ confidence level. After including high-redshift GRBs, the constraints can be tightened to $\Omega_{\mathrm{d}}^{\mathrm{e}}<0.03$ at $2 \sigma$ confidence level.

\subsection{Growth index}

In this section, we put constraint on the growth factor. The observational probe of the growth function $\delta(z)$ is the redshift dependence of the rms mass fluctuation $\sigma_{8}(z)$ defined by

$\sigma^{2}(R, z)=\int_{0}^{\infty} W^{2}(k R) \Delta^{2}(k, z) \frac{\mathrm{d} k}{k}$,

with

$W(k R)=3\left(\frac{\sin (k R)}{(k R)^{3}}-\frac{\cos (k R)}{(k R)^{2}}\right) r$,

$\Delta^{2}(k z)=4 \pi k^{3} P_{\delta}(k, z)$,

with $R=8 h^{-1} \mathrm{Mpc}$ and $P_{\delta}(k, z)$ the mass power spectrum at redshift $z$. The function $\sigma_{8}(z)$ is

$\sigma_{8}(z)=\frac{\delta(z)}{\delta(0)} \sigma_{8}(z=0)$ 


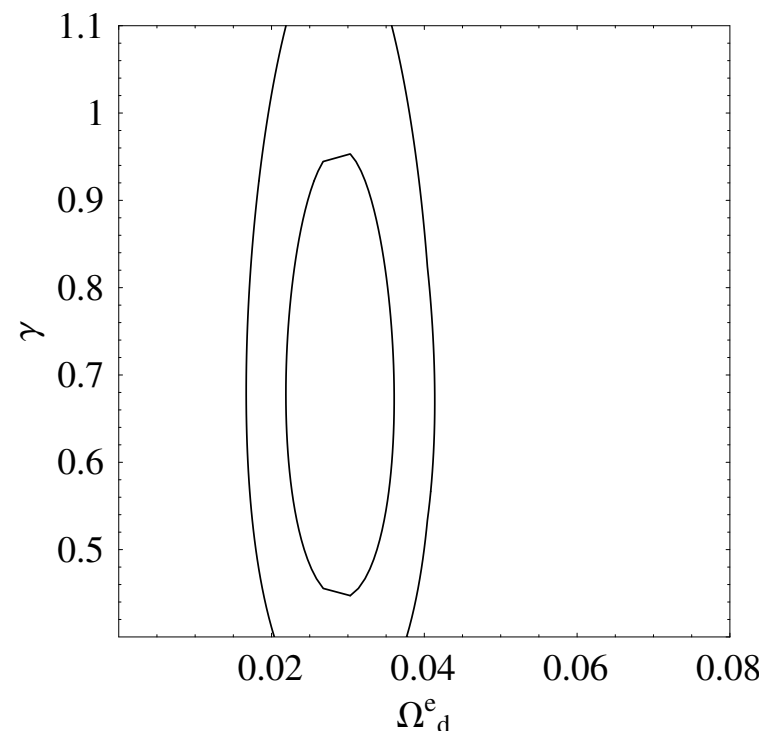

Fig. 8. The $1 \sigma, 2 \sigma$ confidence regions for $\gamma$ versus $\Omega_{\mathrm{d}}^{\mathrm{e}}$ derived using $\mathrm{SNe} \mathrm{Ia}, \mathrm{CMB}, \mathrm{BAO}$ and the linear growth factor data.

which implies

$$
s_{\mathrm{th}}\left(z_{1}, z_{2}\right) \equiv \frac{\sigma_{8}\left(z_{1}\right)}{\sigma_{8}\left(z_{2}\right)}=\frac{\delta\left(z_{1}\right)}{\delta\left(z_{2}\right)}=\frac{e^{\int_{1}^{\frac{1}{1+z_{1}}}} \Omega_{\mathrm{m}}^{\gamma}(a) \frac{\mathrm{d} a}{a}}{e^{\int_{1}^{\frac{1}{1+z_{2}}}} \Omega_{\mathrm{m}}^{\gamma}(a) \frac{\mathrm{d} a}{a}},
$$

where we use Eq. (5). The currently available data points $\sigma_{8}\left(z_{i}\right)$ originate from the observed redshift evolution of the flux power spectrum of Ly $\alpha$ forest (Viel et al. 2004; Viel \& Haehnelt 2006). These data points are shown in Table 1 along with the corresponding references. The currently available data for the parameters $f$ at various redshifts are also shown in Table 1 below.

Using the data of Table 1 we construct the corresponding $\chi_{\text {tot }}^{2}$ defined as

$$
\begin{aligned}
\chi_{\gamma}^{2}(\gamma)= & \sum_{i}\left[\frac{s_{\mathrm{obs}}\left(z_{i}, z_{i+1}\right)-s_{\mathrm{th}}\left(z_{i}, z_{i+1}\right)}{\sigma_{s_{\mathrm{obs}, \mathrm{i}}}}\right]^{2} \\
& +\sum_{i}\left[\frac{f_{\mathrm{obs}}\left(z_{i}\right)-f_{\mathrm{th}}\left(z_{i}\right)}{\sigma_{f_{\mathrm{obs}, \mathrm{i}}}}\right]^{2} \\
& +\sum_{i}\left[\frac{f_{\mathrm{obs}} \sigma_{8}\left(z_{i}\right)-f_{\mathrm{th}} \sigma_{8}\left(z_{i}\right)}{\sigma_{\sigma_{8} f_{\mathrm{obs}, \mathrm{i}}}}\right]^{2}
\end{aligned}
$$

where $\sigma_{s_{\mathrm{obs}, \mathrm{i}}}$ is derived by error propagation from the corresponding $1 \sigma$ errors of $\sigma_{8}\left(z_{i}\right)$ and $\sigma_{8}\left(z_{i+1}\right)$ while $s_{\mathrm{th}}\left(z_{i}, z_{i+1}\right)$ is defined in Eq. (34).

Using the data points in Table 1 and the datasets above, we find $\gamma=0.661_{-0.203}^{+0.302}(1 \sigma)$ with $\chi_{\text {tot,min }}^{2}=\chi_{\mathrm{SN}}^{2}+\chi_{\mathrm{CMB}}^{2}+\chi_{\mathrm{BAO}}^{2}+\chi_{\gamma}^{2}=$ 559.2 by minimizing $\chi_{\text {tot }}^{2}$. This result is a little different from (Xia \& Viel 2009; Nesseris \& Perivolaropoulos 2008; Di Porto \& Amendola 2008), because we have used the new parametrization of early dark energy and latest data set. In Fig. 8, we show the confidence contours of $\gamma$ vs. $\Omega_{\mathrm{d}}^{\mathrm{e}}$. After including high-redshift GRB data, the growth index becomes $\gamma=0.653_{-0.363}^{+0.372}$. We must notice that the $f_{\text {obs }}$ in Table 1 were derived by assuming $\Lambda$ CDM with $\Omega_{\mathrm{m}}=0.30$ when converting redshifts to distances for the power spectra. So their use to test models largely different from $\Lambda$ CDM might be unreliable, as discussed in Nesseris \& Perivolaropoulos (2008). We can use these data when small deviations from flat $\Lambda \mathrm{CDM}$ with $\Omega_{\mathrm{m}}=0.30$ are considered. We

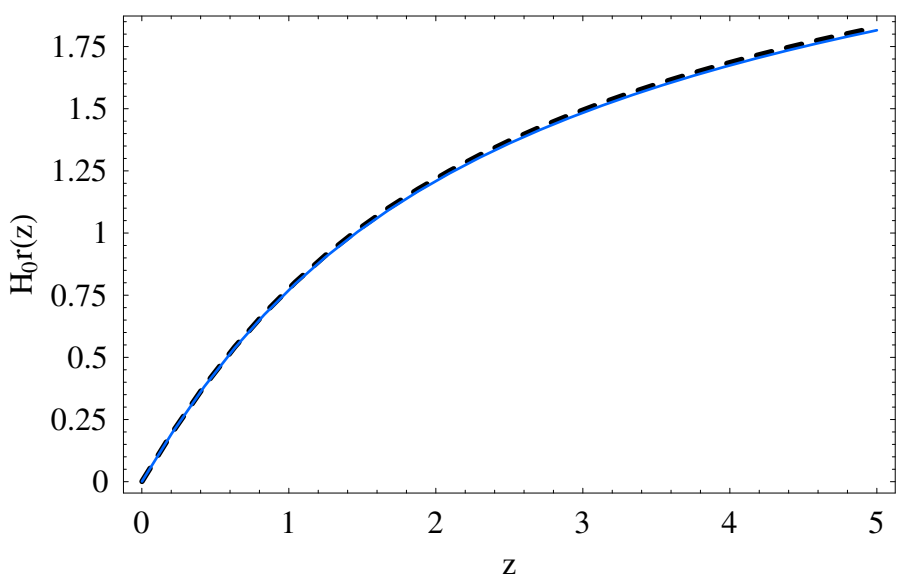

Fig. 9. The solid line shows the $H_{0} r(z)$ in flat $\Lambda$ CDM with $\Omega_{\mathrm{m}}=0.3$ and dashed line shows the $H_{0} r(z)$ in EDE with best fitted parameters $\left(\Omega_{\mathrm{m}}=0.29, \Omega_{\mathrm{d}}^{\mathrm{e}}=0.029, w_{0}=-1.04\right)$. We can see that the redshiftdistance relation in EDE model has small deviations from $\Lambda$ CDM.

believe that we are in such a situation in this paper. As mentioned above, the key problem is the redshift-distance relation. In Fig. 9, we show the relation between $z$ and $H_{0} r(z)$, where $r(z)$ is the comoving distance. The solid line is the $H_{0} r(z)$ in flat $\Lambda \mathrm{CDM}$ with $\Omega_{\mathrm{m}}=0.3$ and dashed line is the $H_{0} r(z)$ in EDE with best fitted parameters $\left(\Omega_{\mathrm{m}}=0.29, \Omega_{\mathrm{d}}^{\mathrm{e}}=0.029, w_{0}=-1.04\right)$. The redshift-distance relation in EDE model has small deviations from $\Lambda \mathrm{CDM}$. So we can use the data of Table 1 in this early dark energy model.

\section{Conclusions and discussion}

In this paper we present constraints on a particular early dark energy model using the latest observations. We use the early dark energy model proposed by Doran \& Robbers (2006). We find that the fractional dark energy density $\Omega_{\mathrm{d}}^{\mathrm{e}}<0.05$ at $2 \sigma$ confidence level, using the latest Union SNe Ia, the WMAP sevenyear data and baryon acoustic oscillations measurement from the SDSS and 2dFGRS. If we add high-redshift GRBs and linear growth factors data from $\operatorname{Ly} \alpha$ forest data, the constraint is improved to $\Omega_{\mathrm{d}}^{\mathrm{e}}<0.03$ at $2 \sigma$ confidence level. The constraint on the growth rate index $\gamma$ is $\gamma=0.661_{-0.203}^{+0.302}(1 \sigma)$ using the $\mathrm{SNe}$ Ia, $\mathrm{CMB}, \mathrm{BAO}$ and linear growth factor data. After including high-redshift GRB data, the growth index is $\gamma=0.653_{-0.363}^{+0.372}$. This result is clearly consistent at $1 \sigma$ with the value predicated by $\Lambda$ CDM.

High-redshift probes, such as GRBs and Ly $\alpha$ are important in constraining early dark energy models. In this paper, we calibrate the luminosity relations of GRBs by SNe Ia using cosmographic parameters. The derived distance modulus of GRBs are model-independent. These measurements of growth factor, summarized in Table 1, have been obtained in the framework of pure $\Lambda \mathrm{CDM}$ model and are valid only when small deviations from this model are considered. Because the references of Table 1 have assumed $\Lambda \mathrm{CDM}$ with $\Omega_{\mathrm{m}}=0.30$ when converting redshifts to distances for the power spectra. In this early dark energy model with the best fitted parameters, the redshift-distance relation is small derivation from $\Lambda$ CDM with $\Omega_{\mathrm{m}}=0.30$. This can be seen from Fig. 9. So the growth factor data are valid in this early dark energy model.

Early dark energy significantly affects the size of the sound horizon. The sound horizon is a crucial quantity because it is 
employed as a standard ruler in the baryon acoustic oscillation technique for probing cosmology. If the standard ruler is miscalibrated due to ignore early dark energy, the cosmological parameters will be biased. Early dark energy also has been shown to influence the growth of cosmic structures, to change the age of the universe, to have an effect on CMB physics. So probing the early dark energy using future observational data is important.

Acknowledgements. This work is supported by the National Natural Science Foundation of China (grant 11103007) and China Postdoctoral Science Foundation funded projects (grants 20100481117 and 201104521).

\section{References}

Alam, U. 2010, ApJ, 714, 1460

Alam, U., Lukic, Z., \& Bhattacharya, S. 2011, ApJ, 727, 87

Amanullah, R., Lidman, C., Rubin, D., et al. 2010, ApJ, 716, 712

Basilakos, S., \& Perivolaropoulos, L. 2008, MNRAS, 391, 411

Blake, C., Brough, S., Colless, M., et al. 2011, MNRAS, 415, 2876

Calabrese, E., R. de Putter, D. Huterer, et al. 2011a, Phys. Rev. D, 83, 023011

Calabrese, E., D. Huterer, E. V. Linder, et al. 2011b, Phys. Rev. D, 83, 123504

Caldwell, R. R. 2002, Phys. Lett. B, 545, 23

Cucchiara, A., Levan, A. J., Fox, D. B., et al. 2011, ApJ, 736, 7

Dai, Z. G., Liang, E. W., \& Xu, D. 2004, ApJ, 612, L101

da Angela, J., Shanks, T., Croom, S. M., et al. 2008, MNRAS, 383, 565

de Putter, R., Zahn, O., \& Linder, E. V. 2009, Phys. Rev. D, 79, 065033

Di Porto, C., \& Amendola, L. 2008, Phys. Rev. D, 77, 083508

Doran, M., \& Robbers, G. 2006, JCAP, 0606, 026

Doran, M., Stern, S., \& Thommes, E. 2007, JCAP, 04, 015

Eisenstein, D. J., \& Hu, W. 1998, ApJ, 496, 605

Eisenstein, D. J., Zehavi, I., Hogg, D. W., et al. 2005, ApJ, 633, 560

Feng, B., Wang, X. L., \& Zhang, X. M. 2005, Phys. Lett. B, 607, 35

Francis, M. J., Lewis, G. F., \& Linder, E. V. 2008, MNRAS, 393, L31 Fry, J. N. 1985, Phys. Lett. B, 158, 211

Gong, Y. G., Ishak, M., \& Wang, A. Z. 2009, Phys. Rev. D, 80, 023002

Grossi, M., \& Springel, V. 2009, MNRAS, 354, 1509

Hawkins, E., Maddox, S., Cole, S., et al. 2003, MNRAS, 346, 78

Hollenstein, L., et al. 2009, JCAP, 0904, 012

Hu, W., \& Sugiyama, N. 1996, ApJ, 471, 542
Izzo, L., Capozziello, S., Covone, G., \& Capaccioli, M. 2009 [arXiv:0906.4888]

Komatsu, E., Smith, K. M., Dunkley, J., et al. 2011, ApJS, 192, 18

Liang, N., et al. 2008, ApJ, 685, 354

Lightman, A. P., \& Schechter, P. L. 1990, ApJ, 74, 831

Linder, E. V. 2005, Phys. Rev. D, 72, 043529

Linder, E. V. 2006, Astropart. Phys., 26, 16

Linder, E. V. 2009, Phys. Rev. D, 79, 063519

Linder, E. V., \& Cahn, R. N. 2007, Astropart. Phys., 28, 481

Linder, E. V., \& Robbers, G. 2008, JCAP, 06, 004

McDonald, P., Seljak, U., Cen, R., et al. 2005, ApJ, 635, 761

Mota, D. F. 2008, JCAP, 09, 006

Nesseris, S., \& Perivolaropoulos, L. 2005, Phys. Rev. D, 72, 123519

Nesseris, S., \& Perivolaropoulos, L. 2008, Phys. Rev. D, 77, 023504

Peebles, P. J. E. 1980, The Large-Scale Structure of the Universe (Princeton, New Jersey: Princeton University Press).

Peebles, P. J. E., \& Ratra, B. 2003, Rev. Mod. Phys., 75, 559

Percival, W. J., Reid, B. A., Eisenstein, D. J., et al. 2010, MNRAS, 401, 2148

Perlmutter, S., Aldering, G., Goldhaber, G., et al. 1999, ApJ, 517, 565

Ratra, B., \& Peebles, P. J. E. 1988, Phys. Rev. D, 37, 3406

Reichardt, C. L., de Putter, R., Zahn, O., \& Hou, Z. 2012, ApJ, 749, L9

Riess, A. G., Filippenko, A. V., Challis, P., et al. 1998, AJ, 116, 1009

Riess, A. G., Macri, L., Casertano, S., et al. 2009, ApJ, 699, 539

Ross, N. P., et al. 2007, MNRAS, 381, 57

Schaefer, B. E. 2007, ApJ, 660, 16

Silveira, V., \& Waga, I. 1994, Phys. Rev. D, 50, 4890

Tegmark, M., et al. 2006, Phys. Rev. D, 74, 123507

Verde, L., et al. 2002, MNRAS, 335, 432

Vitagliano, V., Xia, J. Q., Liberati, S., \& Viel, M. 2010, JCAP, 03, 005

Viel, M., \& Haehnelt, M. G. 2006, MNRAS, 365, 231

Viel, M., Haehnelt, M. G., \& Springel, V. 2004, MNRAS, 354, 684

Wang, Y. 2008, Phys. Rev. D, 78, 123532

Wang, F. Y., \& Dai, Z. G. 2011, A\&A, 536, A96

Wang, L., \& Steinhardt, P. J. 1998, ApJ, 508, 483

Wang, F. Y., Dai, Z. G., \& Zhu, Z. H. 2007, ApJ, 667, 1

Wang, F. Y., Dai, Z. G., \& Qi, S. 2009, RA\&A, 9, 547

Wang, F. Y., Qi, S., \& Dai. Z. G. 2011, MNRAS, 415, 3423

Weinberg, S. 1989, Rev. Mod. Phys., 61, 1

Wetterich, C. 1988, Nucl. Phys. B, 302, 645

Wetterich, C. 2004, Phys. Lett. B, 594, 17

Xia, J.-Q., \& Viel, M. 2009, JCAP, 04, 002

Xia, J. Q., Vitagliano, V., Liberati, S., \& Viel, M. 2012, Phys. Rev. D, 85, 043520 